\title{
ON DESCRIPTION OF GENERALIZED INVARIANT MANIFOLDS FOR NONLINEAR EQUATIONS
}

\author{
A.R. KHAKIMOVA
}

\begin{abstract}
In the paper we discuss the problem on constructing generalized invariant manifolds for nonlinear partial differential equations. A generalized invariant manifold for a given nonlinear equation is a differential connection that is compatible with the linearization of this equation. In fact, this concept generalizes symmetry. Examples of generalized invariant manifolds obtained from symmetries are given in the paper. However, there exist generalized invariant manifolds irreducible to symmetries, exactly they are of the greatest interest. Such generalized invariant manifolds allow one to construct effectively Lax pairs, recursion operators, and particular solutions to integrable equations. In the work we present the algorithm for constructing a generalized invariant manifold for a given equation. A complete description of generalized invariant manifolds of order $(2,2)$ is given for the Korteweg-de Vries equation. We describe briefly a method for constructing a Lax pair and a recursion operator by means of the generalized invariant manifolds. As an example, the Korteweg-de Vries equation is considered.
\end{abstract}

Keywords: Lax pair, higher symmetry, invariant manifold, recursion operator.

Mathematics Subject Classification: 35Q51; 35Q53

\section{INTRODUCTION}

The method of constructing particular solutions to nonlinear partial differential equations based on the notion of differential constraint (or invariant manifold) is well known in the literature, see [1], 2]. The matter of the method is to add a compatible equation to a given equation and as a rule, the compatible equation is simpler. Such technique allows one to find particular solutions to a studied equation. In works [3] - [7] there was proposed a scheme for constructing the Lax pairs and recursion operators for integrable partial differential equations based on the use of similar idea. A suitable generalization is to impose a differential constraint not on the equation, but on its linearization. The resulting equation is referred to as a generalized invariant manifold. This notion is discussed in more detail in Section 2 in the present paper, where required definitions are also given. In Section 3 we provide a complete description of the class of the generalized invariant manifolds of orders $(2,0),(2,1)$ and $(2,2)$ for the Korteweg-de Vries equation. We note that the problem on a complete description of such manifolds for nonlinear equations was not studied before. An algorithm of constructing the Lax pair and recursion operator by a known nontrivial generalized invariant manifold is demonstrated in Section 4 at the example of the KdV equation.

KHAKIMOVA A.R. On DESCRIPTION OF GENERALIZED INVARIANT MANIFOLDS FOR NONLINEAR EQUATIONS. (c) Khakimova A.R. 2018.

The research is supported by Russian Science Foundation (project no. 15-11-20007).

Submitted January 15, 2018. 


\section{MAIN DEFINITIONS}

We consider a nonlinear partial differential equation of the form

$$
u_{t}=f\left(x, t, u, u_{x}, u_{x x}, \ldots, u_{k}\right), \quad u_{j}=\frac{\partial^{j} u}{\partial x^{j}} .
$$

We recall that an ordinary differential equation

$$
u_{r}=g\left(x, t, u, u_{x}, u_{x x}, \ldots, u_{r-1}\right)
$$

is called an invariant manifold for equation (1) if it is compatible with (1), that is, if the condition

$$
D_{x}^{r} f-\left.D_{t} g\right|_{(1), 2]}=0
$$

holds. Here $D_{x}$ and $D_{t}$ are the operators of total differentiation with respect to $x$ and $t$, respectively. We note that condition (3) is equivalent to a partial differential equation for the unknown function $g$. Although sometimes this equation can be solved explicitly, in general, to find the function $g$ is a rather complicated problem.

The situation differs noticeably, when one seeks an ordinary differential equation compatible not with a nonlinear equation (1), but with its linearization

$$
U_{t}=\frac{\partial f}{\partial u} U+\frac{\partial f}{\partial u_{x}} U_{x}+\frac{\partial f}{\partial u_{x x}} U_{x x}+\cdots+\frac{\partial f}{\partial u_{k}} U_{k}
$$

We proceed to rigorous definitions. We consider an ordinary differential equation

$$
U_{m}=F\left(x, t, U, U_{x}, U_{x x}, \ldots, U_{m-1} ; u, u_{x}, u_{x x}, \ldots, u_{n}\right),
$$

where $U=U(x, t)$ is the unknown function sought and the function $u=u(x, t)$ is an arbitrary solution to equation (1) involved in (5) as a functional parameter.

Remark 1. The variables $x, t, U, U_{x}, U_{x x}, \ldots, U_{m-1}, u, u_{x}, u_{x x}, \ldots, u_{n}$ in identity (5) are assumed to be free variables taking arbitrary values.

Definition 1. Equation (5) defines a generalized invariant manifold if the condition

$$
D_{x}^{m} U_{t}-\left.D_{t} U_{m}\right|_{11,44,(5)}=0
$$

holds true identically for all values of the variables $\left\{u_{j}\right\}, x, t, U, U_{x}, \ldots, U_{m-1}$.

Here the variables $u_{t}, U_{t}$ and their derivatives with respect to $x$ are changed by virtue of equations (1) and (4), while the variables $U_{m}, U_{m+1}, \ldots$ are changed by virtue of equality (5). To emphasize that $u(x, t)$ is an arbitrary solution, we treat the variables $u$, $u_{x}, u_{x x}, \ldots$ as independent ones. In view of this, the problem on finding the function $F\left(x, t, U, U_{x}, U_{x x}, \ldots, U_{m-1} ; u, u_{x}, u_{x x}, \ldots, u_{n}\right)$ becomes overdetermined and can be effectively solved. This fact is supported by numerous examples considered in papers [3]-7]. It shown in these papers that the generalized invariant manifold is an efficient tool for constructing the Lax pair and the recursion operator.

Definition 2. Let an invariant manifold $M$ be defined by equation (5). A pair of numbers $(m, n)$ is called the order of the manifold $M$. The manifold $M$ is said to be trivial if an arbitrary solution of equation (5) reads as

$$
U=\varphi\left(x, t, u, u_{x}, \ldots, u_{s}\right), \quad \text { where } \frac{\partial \varphi}{\partial u_{s}} \neq 0 .
$$

Below we provide two examples of trivial generalized invariant manifolds. One can confirm that the equation

$$
U_{x}=\frac{u_{x x}}{u_{x}} U
$$


defines a generalized invariant manifold for the Korteweg-de Vries equation

$$
u_{t}=u_{x x x}+u u_{x} .
$$

This follows the observation that the general solution to equation (6) can be expressed via the classical symmetry $u_{\tau}=u_{x}$ of the KdV equation as

$$
U=c u_{x}
$$

Therefore, it satisfies the linearized equation.

In the same way one can check that a second order equation

$$
U_{x x}=\frac{3 u_{1}^{2} u_{2}+u_{1} u_{5}-u_{3}^{2}}{u_{1} u_{4}+u_{1}^{3}-u_{2} u_{3}} U_{x}+\frac{u_{3}\left(u_{1}^{2}+u_{4}\right)-u_{2} u_{5}-3 u_{1} u_{2}^{2}}{u_{1} u_{4}+u_{1}^{3}-u_{2} u_{3}} U, \quad u_{n}=\frac{\partial^{n}}{\partial x^{n}} u(x, t)
$$

defines a generalized invariant manifold for equation (7). Its general solution is given by the linear combination $U=c_{1} u_{x}+c_{2} u_{t}$ of two symmetries $u_{\tau}=u_{x}$ and $u_{\tau_{1}}=u_{t}$.

\section{COMPLETE DESCRIPTION OF SECOND ORDER GENERALIZED INVARIANT MANIFOLDS FOR THE KDV EQUATION}

Examples (6) and (8) show that one can easily construct the trivial invariant manifolds by means of classical and higher symmetries for the considered equation. However, it seems impossible to construct the Lax pair and recursion operators using such manifolds. More interesting objects are nontrivial generalized invariant manifolds.

The main result of the present paper is the following theorem.

Theorem 1. Let equation $U_{x x}=F\left(U, U_{x}, u, u_{x}, u_{x x}\right)$ define a generalized invariant manifold for the KdV equation (7). Then it is of the form

$$
U_{x x}=\frac{u_{x}}{2\left(u+c_{3}\right)} U_{x}-\frac{2}{3}\left(u+c_{3}\right) U+\frac{u_{x} \sqrt{9 U_{x}^{2}+6\left(u+c_{3}\right)\left(U^{2}+6 c_{4}\right)}}{6\left(u+c_{3}\right)},
$$

where $c_{3}$ and $c_{4}$ are arbitrary constants.

Equation (7) does not possess the generalized invariant manifolds of the form $U_{x x}=$ $F\left(U, U_{x}, u\right)$ and the form $U_{x x}=F\left(U, U_{x}, u, u_{x}, u_{x x}\right)$ provided $\frac{\partial F}{\partial u_{x x}}$ does not vanish identically.

Proof. To prove the theorem, we employ Definition 1. We linearize equation (7):

$$
U_{t}=U_{x x x}+u U_{x}+u_{x} U
$$

and we seek the generalized invariant manifold as

$$
U_{x x}=F\left(U, U_{x}, u, u_{x}\right)
$$

by the condition

$$
D_{x}^{2} U_{t}-\left.D_{t} F\right|_{[7,(9),(10)}=0
$$

We rewrite identity (11) in an expanded form:

$$
\begin{array}{r}
\left(U_{x x x x x}+u U_{x x x}+3 u_{x} U_{x x}+3 u_{x x} U_{x}+u_{x x x} U-F_{U} U_{t}\right. \\
\left.-F_{U_{x}} U_{x, t}-F_{u} u_{t}-F_{u_{x}} u_{x, t}\right)\left.\right|_{7,, 95,10]}=0 .
\end{array}
$$

In identity (12) we change the variables $u_{t}$ and $u_{x, t}$ by virtue of equation (7), $U_{t}$ and $U_{x, t}$ are replaced by virtue of (9), and $U_{x x x}, U_{x x x x x}$ are replaced by virtue of (10). Finally, we obtain:

$$
\begin{aligned}
\alpha_{1}\left(U, U_{x}, u, u_{x}\right) u_{x x x} u_{x x} & +\alpha_{2}\left(U, U_{x}, u, u_{x}\right) u_{x x x}+\alpha_{3}\left(U, U_{x}, u, u_{x}\right) u_{x x}^{3} \\
& +\alpha_{4}\left(U, U_{x}, u, u_{x}\right) u_{x x}^{2}+\alpha_{5}\left(U, U_{x}, u, u_{x}\right) u_{x x}+\alpha_{6}\left(U, U_{x}, u, u_{x}\right)=0
\end{aligned}
$$

where

$$
\alpha_{1}=3 F_{u_{x} u_{x}},
$$




$$
\begin{aligned}
\alpha_{2}= & U+3 U_{x} F_{U u_{x}}+3 F F_{U_{x} u_{x}}+3 u_{x} F_{u u_{x}}, \\
\alpha_{3}= & F_{u_{x} u_{x} u_{x}}, \\
\alpha_{4}= & 3 U_{x} F_{U u_{x} u_{x}}+3 F_{u u_{x}}+3 F_{U_{x} u_{x}} F_{u_{x}}+3 F F_{U_{x} u_{x} u_{x}}+3 u_{x} F_{u u_{x} u_{x}}, \\
\alpha_{5}= & 3 U_{x} F_{U U_{x}} F_{u_{x}}+3 U_{x} F_{U u}+3 u_{x}^{2} F_{u u u_{x}}+3 u_{x} F_{U_{x} u_{x}} F_{u}+3 F^{2} F_{U_{x} U_{x} u_{x}}+3 F F_{U_{x} u} \\
& +3 U_{x} F_{U} F_{U_{x} u_{x}}+3 U_{x}^{2} F_{U U u_{x}}+3 U_{x}+3 F F_{u_{x}} F_{U_{x} U_{x}}+6 U_{x} F F_{U U_{x} u_{x}}+6 u_{x} U_{x} F_{U u u_{x}} \\
& +6 u_{x} F F_{U_{x} u u_{x}}+3 F F_{U_{x}} F_{U_{x} u_{x}}+3 u_{x} F_{u u}-U F_{U_{x}}+3 F F_{U u_{x}}+3 u_{x} F_{u_{x}} F_{U_{x} u}, \\
\alpha_{6}= & 3 u_{x} F+3 F^{2} F_{U U_{x}}+3 u_{x} F F_{U u}+3 U_{x}^{2} F_{U} F_{U U_{x}}+3 u_{x}^{2} F_{u} F_{U_{x} u}+3 U_{x} F^{2} F_{U U_{x} U_{x}} \\
& +3 u_{x} F^{2} F_{U_{x} U_{x} u}+3 u_{x}^{2} U_{x} F_{U u u}+3 u_{x}^{2} F F_{U_{x} u u}+3 U_{x}^{2} F F_{U U U_{x}}+3 u_{x} U_{x}^{2} F_{U U u} \\
& +3 U_{x} F F_{U U}+F^{3} F_{U_{x} U_{x} U_{x}}+u_{x}^{3} F_{u u u}+3 U_{x} F F_{U_{x}} F_{U U_{x}}+3 u_{x} U_{x} F_{u} F_{U U_{x}} \\
& +3 u_{x} U_{x} F_{U} F_{U_{x} u}+3 u_{x} F F_{U_{x}} F_{U_{x} u}+6 u_{x} U_{x} F F_{U U_{x} u}-u_{x} U F_{U}+3 U_{x} F F_{U} F_{U_{x} U_{x}} \\
& +U_{x}^{3} F_{U U U}+3 u_{x} F F_{u} F_{U_{x} U_{x}}+3 F^{2} F_{U_{x}} F_{U_{x} U_{x}}-u_{x}^{2} F_{u_{x}}-2 u_{x} U_{x} F_{U_{x}} .
\end{aligned}
$$

We observe that the variables $u_{x x}, u_{x x x}$ are treated as independent ones and, therefore, (13) holds if and only if the identities

$$
\alpha_{i}\left(U, U_{x}, u, u_{x}\right)=0, \quad i=\overline{1,6},
$$

are satisfied.

Let us study the system of equations (14). As $i=1$ and $i=3$, we deal with the simplest relations. They yield:

$$
F\left(U, U_{x}, u, u_{x}\right)=F_{1}\left(U, U_{x}, u\right) u_{x}+F_{2}\left(U, U_{x}, u\right) .
$$

Taking into consideration representation (15), we can further split the equations in system (14) with respect to the independent variable $u_{x}$. Comparing like powers of $u_{x}$ in the second equation in system (14), we obtain the following two equations:

$$
\begin{aligned}
& \left(F_{1}\right)_{u}+F_{1}\left(F_{1}\right)_{U_{x}}=0, \\
& U+3 F_{2}\left(F_{1}\right)_{U_{x}}+3 U_{x}\left(F_{1}\right)_{U}=0 .
\end{aligned}
$$

Then we express the function $\left(F_{1}\right)_{u}$ from $(16)$ and the function $F_{2}$ from (17):

$$
\begin{aligned}
& \left(F_{1}\right)_{u}=-F_{1}\left(F_{1}\right)_{U_{x}}, \\
& F_{2}=-\frac{U+3 U_{x}\left(F_{1}\right)_{U}}{3\left(F_{1}\right)_{U_{x}}}, \text { where }\left(F_{1}\right)_{U_{x}} \neq 0 .
\end{aligned}
$$

Indeed, assuming $\left(F_{1}\right)_{U_{x}}=0$ in $(19)$, we are led to the identities

$$
U=0 \quad \text { and } \quad\left(F_{1}(U, u)\right)_{U}=0
$$

following (17). This contradicts to the fact that $U$ is a dynamical variable.

In the other equations in system (14), we change all derivatives of $F_{1}$ with respect to variable $u$ by virtue of (18), exclude the function $F_{2}$ by virtue of $(19)$ and we equate the coefficients at the like powers of the variable $u_{x}$. Thus, in addition to (18), we have extra four equations

$$
\begin{aligned}
\text { 1. } & -\frac{3 U_{x} F_{1}\left(F_{1}\right)_{U U}}{\left(F_{1}\right)_{U_{x}}}-\frac{F_{1}\left(F_{1}\right)_{U}\left(3 U_{x}\left(F_{1}\right)_{U}+U\right)\left(F_{1}\right)_{U_{x} U_{x}}}{\left(F_{1}\right)_{U_{x}}^{3}}+\frac{\left(F_{1}\right)_{U} U}{\left(F_{1}\right)_{U_{x}}}+U_{x} \\
& +\frac{F_{1}\left(U+6 U_{x}\left(F_{1}\right)_{U}\right)\left(F_{1}\right)_{U U_{x}}}{\left(F_{1}\right)_{U_{x}}^{2}}=0
\end{aligned}
$$


2. $\frac{F_{1}\left(F_{1}\right)_{U}\left(\left(3 U_{x}\left(F_{1}\right)_{U}+U\right)\left(F_{1}\right)_{U_{x}}-3 F_{1}\left(F_{1}\right)_{U}\right)}{\left(F_{1}\right)_{U_{x}}^{3}}\left(F_{1}\right)_{U_{x} U_{x}}$

$+3 F_{1}\left(U_{x}-\frac{F_{1}}{\left(F_{1}\right)_{U_{x}}}\right)\left(F_{1}\right)_{U U}+F_{1}\left(\frac{6 F_{1}\left(F_{1}\right)_{U}}{\left(F_{1}\right)_{U_{x}}^{2}}-\frac{U+6 U_{x}\left(F_{1}\right)_{U}}{\left(F_{1}\right)_{U_{x}}}\right)\left(F_{1}\right)_{U U_{x}}$

$+F_{1}-U\left(F_{1}\right)_{U}-U_{x}\left(F_{1}\right)_{U_{x}}=0$,

3. $\left(U_{x}^{3}-\frac{6 F_{1} U_{x}^{2}}{\left(F_{1}\right)_{U_{x}}}\right)\left(F_{1}\right)_{U U U}$

$+\left(\frac{F_{1}\left(9 U_{x}\left(F_{1}\right)_{U}+2 U\right)}{\left(F_{1}\right)_{U_{x}}^{2}}-\frac{U_{x}\left(3 U_{x}\left(F_{1}\right)_{U}-2 U\right)}{\left(F_{1}\right)_{U_{x}}}\right)\left(F_{1}\right)_{U U}$

$+\left(\frac{2 U_{x} F_{1}\left(2 U+9 U_{x}\left(F_{1}\right)_{U}\right)-U_{x}^{2}\left(3 U_{x}\left(F_{1}\right)_{U}+U\right)\left(F_{1}\right)_{U_{x}}}{\left(F_{1}\right)_{U_{x}}^{2}}\right)\left(F_{1}\right)_{U U U_{x}}$

$+\left(\frac{U_{x}\left(3 U_{x}\left(F_{1}\right)_{U}+U\right)^{2}}{3\left(F_{1}\right)_{U_{x}}^{2}}-\frac{2 F_{1}\left(3 U_{x}\left(F_{1}\right)_{U}+U\right)\left(9 U_{x}\left(F_{1}\right)_{U}+U\right)}{3\left(F_{1}\right)_{U_{x}}^{3}}\right)\left(F_{1}\right)_{U U_{x} U_{x}}$

$+\left(\frac{2\left(F_{1}\right)_{U} F_{1}\left(3 U_{x}\left(F_{1}\right)_{U}+U\right)^{2}}{3\left(F_{1}\right)_{U_{x}}^{4}}-\frac{\left(3 U_{x}\left(F_{1}\right)_{U}+U\right)^{3}}{27\left(F_{1}\right)_{U_{x}}^{3}}\right)\left(F_{1}\right)_{U_{x} U_{x} U_{x}}$

$+\frac{3 U_{x}^{2}\left(5 F_{1}-U_{x}\left(F_{1}\right)_{U_{x}}\right)\left(F_{1}\right)_{U U_{x}}\left(F_{1}\right)_{U U}}{\left(F_{1}\right)_{U_{x}}^{2}}-\frac{U}{3\left(F_{1}\right)_{U_{x}}}$

$+\left(\frac{U_{x}^{2}\left(3 U_{x}\left(F_{1}\right)_{U}+U\right)}{\left(F_{1}\right)_{U_{x}}^{2}}-\frac{3 U_{x} F_{1}\left(5 U_{x}\left(F_{1}\right)_{U}+U\right)}{\left(F_{1}\right)_{U_{x}}^{3}}\right)\left(F_{1}\right)_{U_{x} U_{x}}\left(F_{1}\right)_{U U}$

$+\left(\frac{2 U_{x}^{2}\left(3 U_{x}\left(F_{1}\right)_{U}+U\right)}{\left(F_{1}\right)_{U_{x}}^{2}}-\frac{U_{x} F_{1}\left(30 U_{x}\left(F_{1}\right)_{U}+7 U\right)}{\left(F_{1}\right)_{U_{x}}^{3}}\right)\left(F_{1}\right)_{U U_{x}}^{2}$

$+\left(\frac{5 F_{1}\left(3 U_{x}\left(F_{1}\right)_{U}+U\right)\left(9 U_{x}\left(F_{1}\right)_{U}+U\right)}{3\left(F_{1}\right)_{U_{x}}^{4}}\right.$

$\left.-\frac{U_{x}\left(3 U_{x}\left(F_{1}\right)_{U}+U\right)^{2}}{\left(F_{1}\right)_{U_{x}}^{3}}\right)\left(F_{1}\right)_{U U_{x}}\left(F_{1}\right)_{U_{x} U_{x}}$

$+\left(\frac{9 U_{x} F_{1}-6 U_{x} U\left(F_{1}\right)_{U}+18 U_{x}^{2}\left(F_{1}\right)_{U}^{2}-2 U^{2}}{3\left(F_{1}\right)_{U_{x}}^{2}}\right.$

$\left.-\frac{F_{1}\left(F_{1}\right)_{U}\left(5 U+18 U_{x}\left(F_{1}\right)_{U}\right)}{\left(F_{1}\right)_{U_{x}}^{3}}\right)\left(F_{1}\right)_{U U_{x}}$

$+\left(\frac{U^{2}\left(F_{1}\right)_{U}-U F_{1}-9 U_{x}^{2}\left(F_{1}\right)_{U}^{3}-9 U_{x} F_{1}\left(F_{1}\right)_{U}}{3\left(F_{1}\right)_{U_{x}}^{3}}\right.$

$\left.+\frac{3 F_{1}\left(F_{1}\right)_{U}^{2}\left(3 U_{x}\left(F_{1}\right)_{U}+U\right)}{\left(F_{1}\right)_{U_{x}}^{4}}\right)\left(F_{1}\right)_{U_{x} U_{x}}$

$+\left(\frac{\left(3 U_{x}\left(F_{1}\right)_{U}+U\right)^{3}}{9\left(F_{1}\right)_{U_{x}}^{4}}-\frac{F_{1}\left(F_{1}\right)_{U}\left(3 U_{x}\left(F_{1}\right)_{U}+U\right)^{2}}{3\left(F_{1}\right)_{U_{x}}^{5}}\right)\left(F_{1}\right)_{U_{x} U_{x}}^{2}=0$,

4. $G\left(U, U_{x}, u, F_{1},\left(F_{1}\right)_{U},\left(F_{1}\right)_{U_{x}},\left(F_{1}\right)_{u},\left(F_{1}\right)_{U U}, \ldots,\left(F_{1}\right)_{U_{x} U_{x} U_{x} U_{x}}\right)=0$.

We do not provide equation (23) explicitly since it is too cumbersome.

We consider equations (20) and (21). We multiply equation (20) by

$$
\left(3 F_{1}\left(F_{1}\right)_{U}-\left(3 U_{x}\left(F_{1}\right)_{U}+U\right)\left(F_{1}\right)_{U_{x}}\right)
$$


and deduct equation (21) multiplied by $\left(3 U_{x}\left(F_{1}\right)_{U}+U\right)$. This gives:

$$
\frac{3\left(F_{1}\right)_{U}^{2}}{\left(F_{1}\right)_{U_{x}}}+\frac{3 F_{1}\left(F_{1}\right)_{U U}}{\left(F_{1}\right)_{U_{x}}}-\frac{3 F_{1}\left(F_{1}\right)_{U}\left(F_{1}\right)_{U U_{x}}}{\left(F_{1}\right)_{U_{x}}^{2}}-1=0 .
$$

We integrate the obtained identity with respect to variable $U$ and resolve with respect to $\left(F_{1}\right)_{U}$ :

$$
\left(F_{1}\right)_{U}=\frac{\left(F_{1}\right)_{U_{x}}\left(U+F_{3}\right)}{3 F_{1}}, \quad F_{3}=F_{3}\left(U_{x}, u\right) .
$$

The sought function should satisfy all equations (18), (20)-(24). The condition of compatibility of (18) and (24) implies the following identity:

$$
\frac{\partial}{\partial U}\left(F_{1}\right)_{u}-\left.\frac{\partial}{\partial u}\left(F_{1}\right)_{U}\right|_{118,24]}=0 .
$$

Hence, by equations (18) and (24), identity (25) casts into the form

$$
-\frac{\left(F_{1}\right)_{U_{x}}\left(F_{1}\left(F_{3}\right)_{U_{x}}+\left(F_{3}\right)_{u}\right)}{3 F_{1}}=0,
$$

and we conclude that $F_{3}\left(U_{x}, u\right)=c_{1}$, where $c_{1}$ is an arbitrary constant.

In equations (20)-(23) we change all derivatives of the function $F_{1}$ with respect to the variable $U$ are changed by virtue of (24). Then equations (20) and (21) hold identically. Then we express $\left(F_{1}\right)_{U_{x} U_{x} U_{x}}$ from equation $(22)$ :

$$
\begin{aligned}
\left(F_{1}\right)_{U_{x} U_{x} U_{x}} & =\frac{3\left(F_{1}\right)_{U_{x} U_{x}}^{2}}{\left(F_{1}\right)_{U_{x}}}-\frac{3\left(6 F_{1} U_{x}+U^{2}-c_{1}^{2}\right)\left(F_{1}\right)_{U_{x}}^{3}}{U^{2} F_{1}^{2}}+\frac{9\left(F_{1}\right)_{U_{x}}^{2}}{U^{2}} \\
& +\frac{9\left(\left(F_{1}\right)_{U_{x}} U_{x}-F_{1}\right)\left(F_{1}\right)_{U_{x} U_{x}}}{U^{2}}+\frac{3 U_{x}\left(3 F_{1} U_{x}+U^{2}-c_{1}^{2}\right)\left(F_{1}\right)_{U_{x}}^{4}}{U^{2} F_{1}^{3}} .
\end{aligned}
$$

Using the compatibility condition for equations (24) and (26), we arrive at two possible cases:

$$
3 U_{x} F_{1}\left(F_{1}\right)_{U_{x}}+c_{1} U\left(F_{1}\right)_{U_{x}}+U^{2}\left(F_{1}\right)_{U_{x}}-3 F_{1}^{2}=0,
$$

or

$$
c_{1}^{2}\left(F_{1}\right)_{U_{x}}^{3}-3 U_{x} F_{1}\left(F_{1}\right)_{U_{x}}^{3}+3 F_{1}^{2}\left(F_{1}\right)_{U_{x}}^{2}-3 F_{1}^{3}\left(F_{1}\right)_{U_{x} U_{x}}=0 .
$$

Assume that equation (27) holds, then

$$
F_{1}=\frac{U_{x}}{2 F_{4}}+\frac{\sqrt{9 U_{x}^{2}+6 F_{4} U\left(c_{1}+U\right)}}{6 F_{4}}, \quad F_{4}=F_{4}(U, u) .
$$

Substituting identity $(29)$ into equations $(18)$ and $(24)$, we find that

$$
c_{1}=0 \quad \text { and } \quad F_{4}=u+c_{2} .
$$

We confirmed that function (29) satisfies equation (23) provided (30) holds. Hence, in case (27), the sought function $F$ reads as

$$
F=\frac{u_{x} U_{x}}{2\left(u+c_{2}\right)}+\frac{u_{x} \sqrt{9 U_{x}^{2}+6\left(u+c_{2}\right) U^{2}}}{6\left(u+c_{2}\right)}-\frac{2}{3}\left(u+c_{2}\right) U .
$$

Now we consider case (28). We rewrite it as

$$
\left(F_{1}\right)_{U_{x} U_{x}}=\frac{\left(F_{1}\right)_{U_{x}}^{2}\left(c_{1}^{2}\left(F_{1}\right)_{U_{x}}-3 U_{x} F_{1}\left(F_{1}\right)_{U_{x}}+3 F_{1}^{2}\right)}{3 F_{1}^{3}} .
$$

Then, substituting 32 into equation 23 , we obtain

$$
c_{1}=0 \quad \text { or } 3 U_{x} F_{1}+2 U c_{1}+U^{2}=0 .
$$

The second equation in (33) contradicts to identity (24), and this is why we let $c_{1}=0$. 
We observe that as $c_{1}=0$, equation $(28)$ is solved explicitly:

$$
F_{1}=\frac{U_{x}}{2 F_{5}}+\frac{\sqrt{U_{x}^{2}+4 F_{5} F_{6}}}{2 F_{5}}, \quad F_{5}=F_{5}(U, u), F_{6}=F_{6}(U, u) .
$$

We determine the functions $F_{5}$ and $F_{6}$ by equations (18) and (24). We substitute (34) into (18) and (24) to get:

$$
F_{5}=u+c_{3}, \quad F_{6}=\frac{1}{6} U^{2}+c_{4} .
$$

Hence, in case (28) we have

$$
F=\frac{u_{x} U_{x}}{2\left(u+c_{3}\right)}+\frac{u_{x} \sqrt{9 U_{x}^{2}+6\left(u+c_{3}\right)\left(U^{2}+6 c_{4}\right)}}{6\left(u+c_{3}\right)}-\frac{2}{3}\left(u+c_{3}\right) U,
$$

where $c_{3}$ and $c_{4}$ are arbitrary constants. Therefore, (31) and (35) represent two nonlinear generalized invariant manifolds. However, we note that (31) is a particular case of (35) and, therefore, equation (11) possesses in fact just one solution

$$
U_{x x}=\frac{u_{x} U_{x}}{2\left(u+c_{3}\right)}+\frac{u_{x} \sqrt{9 U_{x}^{2}+6\left(u+c_{3}\right)\left(U^{2}+6 c_{4}\right)}}{6\left(u+c_{3}\right)}-\frac{2}{3}\left(u+c_{3}\right) U,
$$

which depends on two arbitrary constants $c_{3}, c_{4}$. The proof is complete.

Corollary 1. Generalized invariant manifold (36) is nontrivial.

Suppose that an arbitrary solution of equation $(36)$ is of the form

$$
U=\varphi\left(x, t, u, u_{x}, \ldots, u_{j}\right), \quad \text { where } \quad \frac{\partial \varphi}{\partial u_{j}} \neq 0 .
$$

One can find $U_{x}$ and $U_{x x}$ from equation (37):

$$
\begin{aligned}
& U_{x}=\varphi_{u} u_{x}+\varphi_{u_{x}} u_{x x}+\cdots+\varphi_{u_{j}} u_{j+1}, \\
& U_{x x}=\varphi_{u u} u_{x}^{2}+\varphi_{u} u_{x x}+\cdots+\varphi_{u_{j}} u_{j+2} .
\end{aligned}
$$

In equation (36), we change the functions $U, U_{x}, U_{x x}$ by virtue of identities (37), (38) and (39):

$$
\begin{aligned}
& \varphi_{u u} u_{x}^{2}+\varphi_{u} u_{x x}+\cdots+\varphi_{u_{j}} u_{j+2}=\frac{u_{x}}{2\left(u+c_{3}\right)}\left(\varphi_{u} u_{x}+\varphi_{u_{x}} u_{x x}+\cdots+\varphi_{u_{j}} u_{j+1}\right) \\
& +\frac{u_{x} \sqrt{9\left(\varphi_{u} u_{x}+\varphi_{u_{x}} u_{x x}+\cdots+\varphi_{u_{j}} u_{j+1}\right)^{2}+6\left(u+c_{3}\right)\left(\varphi^{2}+6 c_{4}\right)}}{6\left(u+c_{3}\right)}-\frac{2}{3}\left(u+c_{3}\right) \varphi .
\end{aligned}
$$

Equating the coefficients at higher derivative $u_{j+2}$ in $(40)$, we obtain the identity

$$
\varphi_{u_{j}}=0
$$

which contradicts to assumption (37).

\section{RELATION BETWEEN GENERALIZED INVARIANT MANIFOLDS, THE LAX PAIRS AND RECURSION OPERATORS}

Invariant manifolds are defined by ordinary differential equations depending on constant parameters. To these equations, we can apply the standard ways of order decreasing by finding the integrals or order increasing excluding the constant parameters. We apply one of such transformations to above found manifold (36). Excluding the parameter $c_{4}$ from equation (36) and its differential implication, we arrive to a third order generalized invariant manifold linear in $U, U_{x}, U_{x x}$. Indeed, we rewrite (36) as

$$
U_{x x}-\frac{u_{x} U_{x}}{2\left(u+c_{3}\right)}+\frac{2}{3}\left(u+c_{3}\right) U=\frac{u_{x} \sqrt{9 U_{x}^{2}+6\left(u+c_{3}\right)\left(U^{2}+6 c_{4}\right)}}{6\left(u+c_{3}\right)} .
$$


we calculate the square of both sides of identity (41) and resolve the resulting expression with respect to the constant $c_{4}$ :

$$
c_{4}=\frac{u+c_{3}}{u_{x}^{2}} U_{x x}^{2}+\left(\frac{4\left(u+c_{3}\right)^{2}}{3 u_{x}^{2}} U-\frac{1}{u_{x}} U_{x}\right) U_{x x}-\frac{2\left(u+c_{3}\right)}{3 u_{x}} U U_{x}+\left(\frac{4\left(u+c_{3}\right)^{3}}{9 u_{x}^{2}}-\frac{1}{6}\right) U^{2} .
$$

We differentiate identity 42 with respect to the variable $x$ :

$$
\begin{aligned}
& \left(\frac{2\left(u+c_{3}\right)}{u_{x}^{2}} U_{x x}-\frac{1}{u_{x x}} U_{x}+\frac{4\left(u+c_{3}\right)^{2}}{3 u_{x}^{2}} U\right) U_{x x x}-\frac{2\left(u+c_{3}\right) u_{x x}}{u_{x}^{3}} U_{x x}^{2}-\frac{2\left(u+c_{3}\right)}{3 u_{x}} U_{x}^{2} \\
& -\left(\frac{8\left(u+c_{3}\right) u_{x x}}{3 u_{x}^{3}}-\frac{2\left(u+c_{3}\right)}{u_{x}}\right) U U_{x x}+\left(\frac{2\left(u+c_{3}\right) u_{x x}}{3 u_{x}^{2}}+\frac{8\left(u+c_{3}\right)^{3}}{9 u_{x}^{2}}-1\right) U U_{x} \\
& +\left(\frac{u_{x x}}{u_{x}^{2}}+\frac{4\left(u+c_{3}\right)^{2}}{3 u_{x}^{2}}\right) U_{x} U_{x x}+\left(\frac{4\left(u+c_{3}\right)^{2}}{3 u_{x}}-\frac{8\left(u+c_{3}\right)^{3} u_{x x}}{9 u_{x}^{3}}\right) U^{2}=0 .
\end{aligned}
$$

We simplify the obtained expression by rewriting it in the following form:

$$
\begin{aligned}
& \left(\frac{2\left(u+c_{3}\right)}{u_{x}^{2}} U_{x x}-\frac{1}{u_{x x}} U_{x}+\frac{4\left(u+c_{3}\right)^{2}}{3 u_{x}^{2}} U\right) \\
& \cdot\left(U_{x x x}-\frac{u_{x x}}{u_{x}} U_{x x}+\frac{2}{3}\left(u+c_{3}\right) U_{x}-\left(\frac{2\left(u+c_{3}\right) u_{x x}}{3 u_{x}}-u_{x}\right) U\right)=0 .
\end{aligned}
$$

This equation is split into the following two equations

$$
\begin{aligned}
& \text { 1. } \frac{2\left(u+c_{3}\right)}{u_{x}^{2}} U_{x x}-\frac{1}{u_{x x}} U_{x}+\frac{4\left(u+c_{3}\right)^{2}}{3 u_{x}^{2}} U=0, \\
& \text { 2. } U_{x x x}-\frac{u_{x x}}{u_{x}} U_{x x}+\frac{2}{3}\left(u+c_{3}\right) U_{x}-\left(\frac{2\left(u+c_{3}\right) u_{x x}}{3 u_{x}}-u_{x}\right) U=0 .
\end{aligned}
$$

Assume that condition (43) holds. Then, taking into consideration (41), we have the identity

$$
\left(\frac{1}{u_{x x}}-\frac{1}{u_{x}}\right) U_{x}-\frac{\sqrt{9 U_{x}^{2}+6\left(u+c_{3}\right)\left(U^{2}+6 c_{4}\right)}}{3 u_{x}}=0
$$

which contradicts to the fact that the variables $U_{x}, U, u, u_{x}, u_{x x}, c_{3}, c_{4}$ are free with arbitrary values, see Remark 1 in Section 2. Hence, only (44) holds and this means that

$$
U_{x x x}=\frac{u_{x x}}{u_{x}} U_{x x}-\frac{2}{3}\left(u+c_{3}\right) U_{x}+\left(\frac{2\left(u+c_{3}\right) u_{x x}}{3 u_{x}}-u_{x}\right) U .
$$

A surprising fact is that the resulting third order equation turns out to be linear.

We observe that equations (36) and (45) provide different representations of the same object. The transfer from one writing to the other is made by a simple transformation in the class of ordinary differential equations. However, these representations differ from the point of view of their applications. It is convenient to use the formula (36) to construct the Lax pair, while the equation (45) is more suitable for constructing the recursion operator.

We recall that the recursion operator and the Lax pair are important attributes of the integrability theory. The methods of constructing the Lax pair were discussed earlier in papers [8]-[15]. For the recursion operator we refer for instance, to review [16]. The method of constructing these objects by means of generalized invariant manifold discussed in the present paper was developed in papers [3]-[7].

Let us find the Lax pair for the KdV equation by using the formula (36). Suppose $c_{4}=0$, then the radicand is a quadratic form of $U, U_{x}$ with the coefficient depending on $u, c_{3}$. We rewrite the linearized equation (9) taking into consideration equation (36):

$$
U_{t}=\frac{u_{x x} \sqrt{9 U_{x}^{2}+6\left(u+c_{3}\right) U^{2}}}{6\left(u+c_{3}\right)}+\left(\frac{u_{x x}}{2\left(u+c_{3}\right)}+\frac{u-2 c_{3}}{3}\right) U_{x} .
$$


We observe that the pair of equations (36) and (46) is the Lax pair for KdV equation (7), but it is represented in a nonlinear form. The experience suggests that such obtained Lax pair can be linearized by an appropriate change of variables, cf. [6]. To reduce this pair to a linear form, we express the variables $U$ and $U_{x}$ as some quadratic forms of new variables $\varphi$ and $\psi$ so that the radicand in equation (36) becomes the perfect square. We employ the following lemma, see [6].

Lemma 1. The quadratic form $w(P, Q)=P^{2}+Q^{2}$, where

$$
P=\alpha_{1} \varphi^{2}+\beta_{1} \varphi \psi+\gamma_{1} \psi^{2}, \quad Q=\alpha_{2} \varphi^{2}+\beta_{2} \varphi \psi+\gamma_{2} \psi^{2},
$$

can be written as $P^{2}+Q^{2}=\left(\alpha_{3} \varphi+\beta_{3} \psi\right)^{2}$ if and only if the coefficients of form (47) satisfy one of the following two conditions:

1. $\beta_{2}=\alpha_{1}=\gamma_{1}=0, \beta_{1}^{2}+4 \alpha_{2} \gamma_{2}=0$;

2. there exists a function $h$ such that $\alpha_{2}=h \alpha_{1}, \gamma_{2}=h \gamma_{1}, \beta_{1}=-h \beta_{2}, \beta_{2}^{2}+4 \alpha_{1} \gamma_{1}=0$.

Following Lemma 1, we choose the change of variables

$$
U=\frac{2}{\sqrt{6}} \varphi \psi, \quad U_{x}=\frac{1}{3} \sqrt{u+c_{3}}\left(\varphi^{2}-\psi^{2}\right) .
$$

Then we obtain two systems of equations

$$
\begin{aligned}
& \left\{\begin{array}{l}
\varphi_{x}=\frac{u_{x}}{4\left(u+c_{3}\right)} \varphi-\frac{\sqrt{u+c_{3}}}{\sqrt{6}} \psi \\
\psi_{x}=\frac{\sqrt{u+c_{3}}}{\sqrt{6}} \varphi-\frac{u_{x}}{4\left(u+c_{3}\right)} \psi
\end{array}\right. \\
& \left\{\begin{array}{l}
\varphi_{t}=\left(\frac{3 u_{x x x}+u_{x}\left(u-2 c_{3}\right)}{12\left(u+c_{3}\right)}\right) \varphi-\frac{\sqrt{6}}{18}\left(u-2 c_{3}\right) \sqrt{u+c_{3}} \psi, \\
\psi_{t}=\left(\frac{u_{x x}}{\sqrt{6} \sqrt{u+c_{3}}}+\frac{\sqrt{6}}{18}\left(u-2 c_{3}\right) \sqrt{u+c_{3}}\right) \varphi-\left(\frac{3 u_{x x x}+u_{x}\left(u-2 c_{3}\right)}{12\left(u+c_{3}\right)}\right) \psi
\end{array}\right.
\end{aligned}
$$

instead of the pair of equations (36), (46). Pair (48), (49) represents the linear Lax pair for KdV equation (7). Let us reduce pair (48), (49) to a known Lax pair. To exclude the dependence on $u_{x}$ in system (48), we employ the change of variables $\varphi=\alpha p$ and $\psi=\beta q$ that reduces (48) to the form

$$
\left\{\begin{array}{l}
p_{x}=\left(\frac{u_{x}}{4\left(u+c_{3}\right)}-\frac{\alpha_{x}}{\alpha}\right) p-\frac{\sqrt{u+c_{3}}}{\sqrt{6}} \frac{\beta}{\alpha} q, \\
q_{x}=\frac{\sqrt{u+c_{3}}}{\sqrt{6}} \frac{\alpha}{\beta} p-\left(\frac{u_{x}}{4\left(u+c_{3}\right)}+\frac{\beta_{x}}{\beta}\right) q .
\end{array}\right.
$$

We postulate the identities

$$
\frac{\alpha_{x}}{\alpha}=\frac{u_{x}}{4\left(u+c_{3}\right)}, \quad \frac{\beta_{x}}{\beta}=-\frac{u_{x}}{4\left(u+c_{3}\right)}
$$

and this implies $\alpha=\left(u+c_{3}\right)^{\frac{1}{4}}, \beta=\left(u+c_{3}\right)^{-\frac{1}{4}}$ and, therefore, 50 becomes

$$
\left\{\begin{array}{l}
p_{x}=-\frac{1}{\sqrt{6}} q, \\
q_{x}=\frac{1}{\sqrt{6}}\left(u+c_{3}\right) p .
\end{array}\right.
$$

From system (51), we proceed to a the second order equation for $p$ :

$$
p_{x x}=-\frac{1}{6}\left(u+c_{3}\right) p .
$$


As a result of the above transformations, system 490 reduces to an equation of the form

$$
p_{t}=\frac{1}{3}\left(u-2 c_{3}\right) p_{x}-\frac{1}{6} u_{x} p
$$

Pair of equations (52), (53) coincides with the known Lax pair for the KdV equation, see [17].

Now let us show how to find the recursion operator for the KdV equation (7) using the generalized invariant manifold (45). We rewrite equation (45) as

$$
U_{x x x}-\frac{u_{x x}}{u_{x}} U_{x x}+\frac{2}{3} u U_{x}-\left(\frac{2 u u_{x x}}{3 u_{x}}-u_{x}\right) U=-\frac{2}{3} c_{3} u_{x} D_{x}\left(\frac{1}{u_{x}} U\right) .
$$

In order to obtain the recursion operator from equation (54), we need to represent this equation in the form $R U=\lambda U$. To do this, the equation (54) is multiplied by operator $u_{x} D_{x}^{-1}\left(\frac{1}{u_{x}}\right)$ and by some straightforward transformations we arrive at the expression

$$
\left(D_{x}^{2}+\frac{2}{3} u+\frac{1}{3} u_{x} D_{x}^{-1}\right) U=\lambda U, \quad \lambda=-\frac{2}{3} c_{3} .
$$

Thus, the required recursion operator is of the form

$$
R=D_{x}^{2}+\frac{2}{3} u+\frac{1}{3} u_{x} D_{x}^{-1} .
$$

Constructed operator (55) coincides with a known recursion operator of the KdV equation, see [18].

The author expresses her deep gratitude to I.T. Habibullin for setting up the problem and permanent attention to this work. She also thanks the participants of the seminar of the Department of High Performance Computing Systems and Technology of USATU supervised by R.K. Gazizov for useful remarks and advices.

\section{BIBLIOGRAPHY}

1. A.F. Sidorov, V.P. Shapeev, N.N. Yanenko. The method of differential constraints and its applications in gas dynamics, Nauka, Novosibirsk (1984). (in Russian)

2. N.N. Yanenko. On invariant differential constraints for hyperbolic systems of quasilinear equations // Izv. Vyssh. Ucheb. Zav. Ser. Matem. 3, 185-194 (1961). (in Russian).

3. I.T. Habibullin, A.R. Khakimova, M.N. Poptsova. On a method for constructing the Lax pairs for nonlinear integrable equations // J. Phys. A. Math. Theor. 49:3, id 035202 (2016).

4. E.V. Pavlova, I.T. Habibullin, A.R. Khakimova On one integrable discrete system // Itogi Nauki Tekhn. Ser. Sovrem. Matem. Pril. 140, 30-42 (2017). (in Russian).

5. I.T. Habibullin, A.R. Khakimova. Invariant manifolds and Lax pairs for integrable nonlinear chains // Teor. Matem. Fiz. 191:3, 369-388 (2017). [Theor. Math. Phys. 191:3, 793-810 (2017).]

6. I.T. Habibullin, A.R. Khakimova. On a method for constructing the Lax pairs for integrable models via a quadratic ansatz // J. Phys. A. Math. Theor. 50:30, id 305206 (2017).

7. I.T. Habibullin, A.R. Khakimova. A direct algorithm for constructing recursion operators and Lax pairs for integrable models // Teor. Matem. Fiz. 196:2, 294-312 (2018). [Theor. Math. Phys. 196:2, 1200-1216 (2018).]

8. V.E. Zakharov, A.B. Shabat. A scheme for integrating the nonlinear equations of mathematical physics by the method of the inverse scattering problem. I // Funkts. Anal. Pril. 8:3, 43-53 (1974). [Funct. Anal. Appl. 8:3, 226-235 (1974).]

9. V.E. Zakharov, A.B. Shabat. Integration of nonlinear equations of mathematical physics by the method of inverse scattering. II // Funkts. Anal. Pril. 13:3, 13-22 (1979). [Funct. Anal. Appl. 13:3, 166-174 (1979).]

10. H.D. Wahlquist, F.B. Estabrook. Prolongation structures of nonlinear evolution equations // J. Math. Phys. 16:1, 1-7 (1975). 
11. F.W. Nijhoff, A.J. Walker. The discrete and continuous Painlevé VI hierarchy and the Garnier system // Glasgow Math. J. 43:A, 109-123 (2001).

12. A.I. Bobenko, Yu.B. Suris. Integrable systems on quad-graphs // Int. Math. Res. Notes 2002:11, 573-611 (2002).

13. F.W. Nijhoff. Lax pair for the Adler (lattice Krichever-Novikov) system // Phys. Lett. A. 297:1-2, 49-58 (2002).

14. R.I. Yamilov. On classification of discrete equations // in "Integrable systems", ed. A. B. Shabat, Bashkir Branch AS USSR, Ufa. 95-114 (1982). (in Russian)

15. P. Xenitidis. Integrability and symmetries of difference equations: the Adler-Bobenko-Suris case // in "Group Analysis of Differential Equations and Integrable Systems" Proc. 4th Workshop, 226-242 (2009).

16. V.V. Sokolov. Algebraic structures related to integrable differential equations // Ensaios Matemáticos. 31, 1-108 (2017).

17. P.D. Lax. Integrals of nonlinear equations of evolution and solitary waves // Comm. Pure Appl. Math. 21:5, 467-490 (1968).

18. C.S. Gardner, J.M. Greene, M.D. Kruskal, R.M. Miura. Korteweg-de Vries equation and generalizations. VI. methods for exact solution // Comm. Pure Appl. Math. 27:1, 97-133 (1974).

Aigul Rinatovna Khakimova,

Bashkir State University,

Zaki Validi str. 32,

450077, Ufa, Russia

Institute of Mathematics,

Ufa Federal Research Center, RAS,

Chernyshevsky str. 112,

450008, Ufa, Russia

E-mail: aigulya.khakimova@mail.ru 